PROCEEDINGS OF THE

AMERICAN MATHEMATICAL SOCIETY

Volume 139, Number 3, March 2011, Pages 975-984

S 0002-9939(2010)10616-0

Article electronically published on July 30,2010

\title{
NONDEGENERACY \\ OF THE SECOND BIFURCATING BRANCHES \\ FOR THE CHAFEE-INFANTE PROBLEM \\ ON A PLANAR SYMMETRIC DOMAIN
}

\author{
YASUHITO MIYAMOTO
}

(Communicated by Matthew J. Gursky)

\begin{abstract}
Let $\Omega$ be a planar domain such that $\Omega$ is symmetric with respect to both the $x$ - and $y$-axes and $\Omega$ satisfies certain conditions. Then the second eigenvalue of the Dirichlet Laplacian on $\Omega, \nu_{2}(\Omega)$, is simple, and the corresponding eigenfunction is odd with respect to the $y$-axis. Let $f \in C^{3}$ be a function such that$$
f^{\prime}(0)>0, f^{\prime \prime \prime}(0)<0, f(-u)=-f(u) \text { and } \frac{d}{d u}\left(\frac{f(u)}{u}\right)<0 \text { for } u>0 .
$$

Let $\mathcal{C}$ denote the maximal continua consisting of nontrivial solutions, $\{(\lambda, u)\}$, to

$$
\Delta u+\lambda f(u)=0 \text { in } \Omega, \quad u=0 \text { on } \partial \Omega
$$

and emanating from the second eigenvalue $\left(\nu_{2}(\Omega) / f^{\prime}(0), 0\right)$. We show that, for each $(\lambda, u) \in \mathcal{C}$, the Morse index of $u$ is one and zero is not an eigenvalue of the linearized problem. We show that $\mathcal{C}$ consists of two unbounded curves, each curve is parametrized by $\lambda$ and the closure $\overline{\mathcal{C}}$ is homeomorphic to $\mathbb{R}$.
\end{abstract}

\section{INTRODUCTION AND MAIN RESULT}

Let $\Omega$ be a planar domain with smooth boundary. In this paper we study the Dirichlet problem

$\left(\mathrm{DP}_{\Omega}\right) \quad \Delta u+\lambda f(u)=0$ in $\Omega, \quad u=0$ on $\partial \Omega$,

where we write $\Omega$ in $\left(\mathrm{DP}_{\Omega}\right)$ in order to specify the dependence of the problem on the domain. We assume that $f$ satisfies

$$
\begin{gathered}
f \in C^{3}, f^{\prime}(0)>0, f^{\prime \prime \prime}(0)<0, f(-u)=-f(u) \text { and } \\
\frac{d}{d u}\left(\frac{f(u)}{u}\right)<0 \text { for } u>0 .
\end{gathered}
$$

A typical example is the Allen-Cahn equation $\Delta u+\lambda\left(u-u^{3}\right)=0$. Let $\left\{\nu_{n}(\Omega)\right\}_{n=1}^{\infty}$ $\left(\nu_{1}(\Omega)<\nu_{2}(\Omega) \leq \nu_{3}(\Omega) \leq \cdots\right)$ denote the eigenvalues, counting multiplicities, of

Received by the editors March 23, 2010.

2010 Mathematics Subject Classification. Primary 35B32, 35P15; Secondary 35J61, 35J15.

Key words and phrases. Global branch, bifurcation, sign-changing solution, planar symmetric domain, second eigenvalue.

This work was partially supported by Grant-in-Aid for Young Scientists (B) (Subject No. 21740116).

(C)2010 American Mathematical Society Reverts to public domain 28 years from publication 


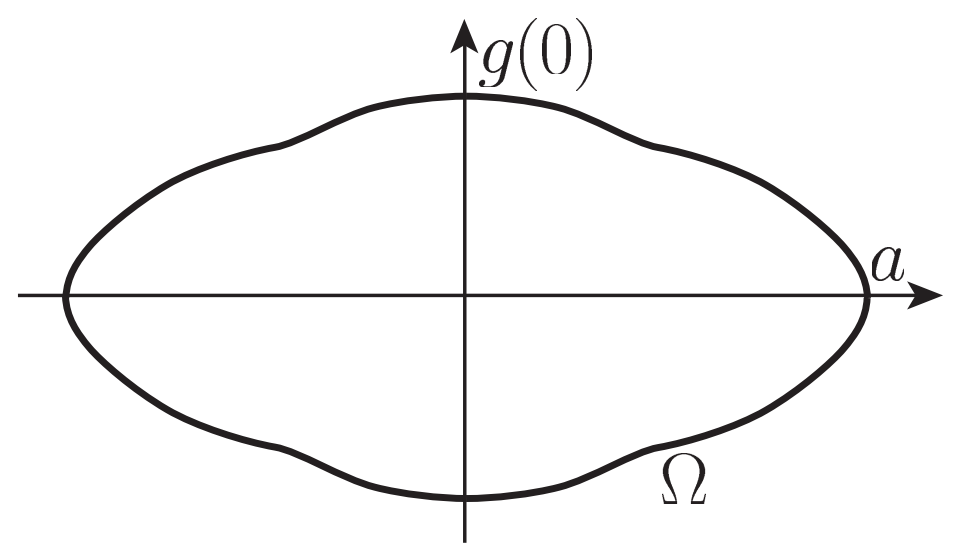

Figure 1. An example of the domain $\Omega$ satisfying (D1)-(D3)

the Laplacian with the Dirichlet boundary condition, $\Delta_{D}$, on $\Omega$. We assume that $\Omega$ satisfies the following:

(D1)

$g \in C^{1}([0, a)) \cap C^{0}([0, a])$ and $\bar{\Omega}=\{(x, y) ;-a \leq x \leq a,-g(|x|) \leq y \leq g(|x|)\} \subset \mathbb{R}^{2}$,

$$
\begin{aligned}
g>0 \text { on }[0, a), g(a)=0, g^{\prime} & <0 \text { on }(0, a), g^{\prime}(0)=0, \\
\frac{d}{d x}\left(x^{2}+(g(x))^{2}\right) & <0 \text { on }(0, a) .
\end{aligned}
$$

Shen 14 has shown that (4) stated below follows from (D1)-(D3). See also Pütter [13.

The second eigenvalue of $\Delta_{D}$ on $\Omega, \nu_{2}(\Omega)$, is simple, and the corresponding eigenfunction is odd with respect to the $y$-axis.

An ellipse is a typical example of the domain satisfying (D1)-(D3). Note that the convexity of $\Omega$ is not assumed in (D1) $-(\overline{D 3})$. Figure 1 is an example which is not convex.

Let $X$ be a functional space. We call $\{(\lambda, 0)\} \subset \mathbb{R} \times X$ a trivial solution. Since $\nu_{2}(\Omega)$ is simple, we can apply the Crandall-Rabinowitz bifurcation theorem 3 . We see that the nontrivial solutions of $\left(\mathrm{DP}_{\Omega}\right)$ near $\left(\nu_{2}(\Omega) / f^{\prime}(0), 0\right)$ consist of two curves emanating from $\left(\nu_{2}(\Omega) / f^{\prime}(0), 0\right)$. Because $f^{\prime \prime}(0)=0$ and $f^{\prime \prime \prime}(0)<0$, this bifurcation is supercritical; hence the bifurcating branches are in $\left\{\lambda>\nu_{2}(\Omega) / f^{\prime}(0)\right\}$. The main theorem of the paper is

Theorem A. Suppose that (D1)-(D3) and (E) hold. (Hence (D4) also holds.) The maximal continua, $\mathcal{C}$, consisting of nontrivial solutions to $\left(D P_{\Omega}\right)$ and emanating from the second eigenvalue $\left(\nu_{2}(\Omega) / f^{\prime}(0), 0\right)$ consist of exactly two unbounded curves $\Sigma_{+}$and $\Sigma_{-}$, i.e., $\mathcal{C}=\Sigma_{+} \cup \Sigma_{-}$. Each curve is parametrized by $\lambda$, and the closure $\overline{\mathcal{C}}$ is homeomorphic to $\mathbb{R}$. (See Figure 2.) For each $(\lambda, u) \in \Sigma_{ \pm}$, the Morse index of $u$, which is the number of the negative eigenvalues of

$$
\Delta \varphi+\lambda f^{\prime}(u) \varphi+\kappa \varphi=0 \text { in } \Omega, \quad \varphi=0 \text { on } \partial \Omega,
$$

is one, and $u$ is nondegenerate, i.e., zero is not an eigenvalue of (1.1). 


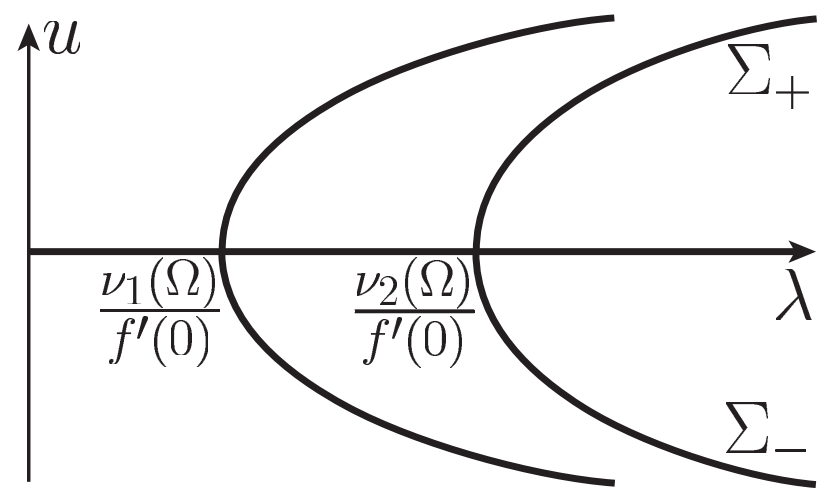

FiguRE 2. A schematic picture of the bifurcation diagram of $\left(\mathrm{DP}_{\Omega}\right)($ Theorem $\mathrm{A})$.

$\Sigma_{+}$and $\Sigma_{-}$are defined by (3.5) in Section 3. The shape of $u \in \Sigma_{ \pm}$is studied in Lemma 3.4. The nondegeneracy stated in Theorem A immediately means that $\Sigma_{+}$ and $\Sigma_{-}$do not have a secondary bifurcation point.

Let us explain a background and known results related to the bifurcation problem $\left(\mathrm{DP}_{\Omega}\right)$. Our problem $\left(\mathrm{DP}_{\Omega}\right)$ with $(\mathrm{F})$ is a high-dimensional version of the ChafeeInfante problem [2. Thus it is expected that under certain conditions on the domain the maximal continua emanating from an eigenvalue

consist of two unbounded curves, each curve is parametrized by $\lambda$ and the closure of the two curves is homeomorphic to $\mathbb{R}$.

It is well-known that if $f$ satisfies $(\mathbb{F})$ and $\Omega$ is arbitrary, then the first bifurcating branches satisfy (1.2). It is conjectured that if $\Omega$ is convex and $\nu_{2}(\Omega)$ is simple, then the second bifurcating branches satisfy (1.2). Theorem $\mathrm{A}$ is a positive answer for a planar domain satisfying (D1) (D3). When $\Omega$ is a disk, the multiplicity of the second eigenvalue is two. In this case the maximal continua consisting of nontrivial solutions and emanating from the second eigenvalue can be parametrized by $(\theta, \lambda) \in$ $S^{1} \times(\underline{\lambda}, \bar{\lambda})(\bar{\lambda} \in(\underline{\lambda},+\infty])$ for the Dirichlet [11] and Neumann [10] problems, it is homeomorphic to $S^{1} \times \mathbb{R}$ for each problem and the closure is homeomorphic to $\mathbb{R}^{2}$ for each problem. Here $S^{1}$ comes from the rotational invariance of the domain. When $\Omega$ is a square, del Pino et al. [4 studied the local bifurcation at the second eigenvalue. They have shown that the closure of the nontrivial solutions locally consists of exactly four curves for certain nonlinear terms including $f(u)=u+u^{3}$. However, the global property is not known. On the contrary, Vegas 15 has shown that if the domain has a dumbbell-like shape (hence nonconvex), the nonlinearity is $\lambda u-a u^{p}+O\left(|u|^{p+1}\right)$ and the Neumann boundary condition is imposed, then another continuum of nontrivial solutions emanates from a branch from the second eigenvalue. Hence the closure of the maximal continua is not homeomorphic to $\mathbb{R}$ (see [15, Figure 3]).

Another direction of generalization is to consider the case where (F) is not imposed and $\Omega$ has certain symmetries. Holzmann-Kielhöfer [6] studied the continuum, $\mathcal{C}_{+}$, of the positive solutions to $\left(\overline{\mathrm{DP}_{\Omega}}\right)$ when $\Omega$ satisfies (D1) and (D2) and $f(u) \geq 0$ for $u \geq 0$. They have shown that $\mathcal{C}_{+}$can be parametrized by $\|u\|_{L^{\infty}}=u(0,0), \mathcal{C}_{+}$may have a turning point, but other nontrivial solutions do 
not bifurcate from $\mathcal{C}_{+}$. When $f(0)=0$ and $f^{\prime}(0)>0,\{(\lambda, 0)\}$ is a trivial solution of $\left(\overline{\mathrm{DP}_{\Omega}}\right)$ and $\mathcal{C}_{+}$emanates from $\left(\nu_{1}(\Omega) / f^{\prime}(0), 0\right)$. When $f(u) \leq 0$ for $u \leq 0$, the continuum of negative solutions obviously has the same property. Thus the closure of the first bifurcating branches is homeomorphic to $\mathbb{R}$. (See the first bifurcating branch of Figure 2 of the present paper and [6, Figures 1 and 3].) Korman [8] showed that a result similar to 6] holds for the positive solutions of $\left(\mathrm{DP}_{\Omega}\right)$ when $\Omega$ is a ball in $\mathbb{R}^{N}$. When $f$ is arbitrary and $\Omega$ is a disk, partial results about the existence of unbounded continua consisting of nonradially symmetric solutions are known for the Dirichlet [11, 12] and Neumann [9] problems.

Let us mention the technique used in the proof of Theorem A. Our main tool is the nodal curve of the eigenfunction. We use the derivative

$$
\partial_{\theta}:=-y \partial_{x}+x \partial_{y} .
$$

By direct calculation we see that $\partial_{\theta}$ commutes with $\Delta\left(=\partial_{x}^{2}+\partial_{y}^{2}\right)$, i.e., $\partial_{\theta} \Delta=\Delta \partial_{\theta}$. Let $u$ be a solution of $\left(\mathrm{DP}_{\Omega}\right)$. Then $u_{\theta}$ satisfies the linear equation $\Delta u_{\theta}+\lambda f^{\prime}(u) u_{\theta}=$ 0 . When the domain is a disk and $u$ is nonradially symmetric, $u_{\theta}(\not \equiv 0)$ satisfies the Dirichlet boundary condition. Thus zero is an eigenvalue and $u_{\theta}$ is an associated eigenfunction. We used this fact in the disk case [11. In the proof of Theorem A we use a contradiction argument. Suppose that zero is the second eigenvalue. Let $\varphi$ be an associated eigenfunction. Then $\varphi$ satisfies $\Delta \varphi+\lambda f^{\prime}(u) \varphi=0$. In our problem $\left(\mathrm{DP}_{\Omega}\right) u_{\theta}$ does not satisfy the Dirichlet boundary condition. We improve the argument used in the disk case [11. Combining $\varphi$ and $u_{\theta}$ (or $u_{x}$ ), we can construct an eigenfunction that has two nodal domains and vanishes on an open set in $\Omega$. (See the proof of Lemma 3.2.) This eigenfunction leads to the contradiction. In other words, we obtain information on the Morse index of $u$ from the shape of $u$.

The paper consists of three sections. In Section 2 we recall the Hartman-Wintner theory [7] which can analyze the nodal curve of the eigenfunction. In Section 3 we prove Theorem A,

\section{Preliminaries}

In Section 3 we use the local property of a solution to a linear equation on a planar domain near a degenerate zero.

Proposition 2.1 ([1, 7]). Let $\Omega$ be a planar domain. Let $V(x, y) \in C^{1}(\Omega)$, and let $u(x, y)$ be a function such that $\Delta u+V u=0$ in $\Omega$. Then $u \in C^{2}(\Omega)$. If $p_{0} \in \Omega$ is a zero of $u$, then one of the followings holds:

(i) $p_{0} \in \Omega$ is a zero of every order and $u \equiv 0$ in $\Omega$.

(ii) There is $n \in \mathbb{N}$ such that the Taylor expansion of $u$ is

$$
u(p)=H_{n}\left(p-p_{0}\right)+o\left(\left|p-p_{0}\right|^{n}\right),
$$

where $H_{n}$ is a real valued, nonzero, harmonic, homogeneous polynomial of degree $n$. Moreover, $\{u=0\}$ has exactly $2 n$ curves near $p_{0}$, and they meet $p_{0}$ with equal angle.

This proposition is due to Hartman-Wintner [7] and generalizes a result of Carleman [1].

The next corollary is an immediate consequence of Proposition 2.1 which is used in Section 3. 
Corollary 2.2. Let $u$ be as mentioned in Proposition 2.1. If $p_{0} \in \Omega$ is a degenerate zero of $u$ of order at least two, i.e., $u\left(p_{0}\right)=u_{x}\left(p_{0}\right)=u_{y}\left(p_{0}\right)=0$, then $u \equiv 0$ in $\Omega$ or $\{u=0\}$ has at least four curves meeting $p_{0}$ with equal angle.

\section{Proof of Theorem A}

In this section, let $\Omega$ be a planar domain such that (D1)-(D3) hold. Then (D4) also holds. We define

$$
\begin{gathered}
S:=\{y=0\}, T:=\{x=0\}, T_{+}:=\{x>0\}, T_{-}:=\{x<0\}, \\
\Omega_{+}:=\Omega \cap T_{+}, \Omega_{-}:=\Omega \cap T_{-}, \Gamma_{+}:=\partial \Omega \cap\{y>0\}, \Gamma_{-}:=\partial \Omega \cap\{y<0\} .
\end{gathered}
$$

Let $f$ be a function such that $(\mathbb{E})$ holds. Without loss of generality, we can assume that

$$
f^{\prime}(0)=1
$$

because of the changing of the variable $\lambda \mapsto \lambda / f^{\prime}(0)$. We always assume (3.1) in this section. The second bifurcation point of $\left(\mathrm{DP}_{\Omega}\right)$ is $\left(\nu_{2}(\Omega), 0\right)$.

We construct two unbounded curves consisting of nontrivial solutions to $\left(\mathrm{DP}_{\Omega}\right)$ and emanating from $\left(\nu_{2}(\Omega), 0\right)$. First, we consider the half problem $\left(\mathrm{DP}_{\Omega_{+}}\right)$. It is well-known that because $f(u) / u$ is decreasing for $u>0$, the positive branch, which emanates from $\left(\nu_{1}\left(\Omega_{+}\right), 0\right)$, is unbounded and can be parametrized by $\lambda$. We briefly prove this. We define $F(\lambda, u):=u+\Delta_{D}^{-1}[\lambda f(u)]$. Let $\left(\lambda_{0}, u_{0}\right)$ be a positive solution of $\left(\mathrm{DP}_{\Omega_{+}}\right)$. Since $f(u) / u$ is decreasing for $u>0, f^{\prime}(u)-\frac{f(u)}{u}=u \frac{d}{d u}\left(\frac{f(u)}{u}\right)<0$ for $u>0$. Because of the comparison of eigenvalues, the first eigenvalue of

$$
\Delta \varphi+\lambda_{0} f^{\prime}\left(u_{0}\right) \varphi+\kappa \varphi=0 \text { in } \Omega_{+}, \quad \varphi=0 \text { on } \partial \Omega_{+}
$$

is greater than

$$
\Delta \varphi+\lambda_{0} V\left(u_{0}\right) \varphi+\kappa \varphi=0 \text { in } \Omega_{+}, \quad \varphi=0 \text { on } \partial \Omega_{+},
$$

where

$$
V(u):= \begin{cases}f(u) / u & \text { if } u \neq 0 \\ f^{\prime}(0) & \text { if } u=0\end{cases}
$$

The first eigenvalue of (3.3) is zero because $(\kappa, \varphi)=\left(0, u_{0}\right)$ satisfies (3.3) and $u_{0}$ does not change sign in $\Omega_{+}$. The first eigenvalue of (3.2) is greater than zero, and zero is not an eigenvalue. Hence $F_{u}\left(\lambda_{0}, u_{0}\right)$ is invertible. Applying the implicit function theorem to $F(\lambda, u)=0$, we obtain a mapping $u=u(\lambda)$ such that $F(\lambda, u(\lambda))=0$ for a neighborhood of $\lambda=\lambda_{0}$. The domain of the mapping $u=u(\lambda)$ may be bounded or unbounded. For example, the domain is bounded (resp. unbounded) if $\lim _{u \rightarrow+\infty} f(u) / u>0($ resp. $<0)$. Let $\left(\nu_{1}\left(\Omega_{+}\right), \bar{\mu}\right)(\bar{\mu}$ may be $+\infty)$ be the maximal domain of $u=u(\lambda)$.

We extend this solution $(\lambda, u(x, y ; \lambda))$ with odd reflection as follows:

$$
u^{*}(x, y ; \lambda):=\left\{\begin{array}{cl}
u(x, y ; \lambda) & \text { if }(x, y) \in \overline{\Omega_{+}}, \\
-u(-x, y ; \lambda) & \text { if }(x, y) \in \overline{\Omega_{-}} .
\end{array}\right.
$$

Then $u^{*}$ is differentiable on $\Omega \cap T$ and $\left(\lambda, u^{*}\right)$ is a classical solution of $\left(\mathrm{DP}_{\Omega}\right)$. Note that the mapping $\lambda \mapsto u^{*}(\lambda)$ is of class $C^{1}$. We denote two curves of nontrivial solutions by

$$
\Sigma_{+}:=\left\{\left(\lambda, u^{*}(\lambda)\right) ; \underline{\mu}<\lambda<\bar{\mu}\right\}, \quad \Sigma_{-}:=\left\{\left(\lambda,-u^{*}(\lambda)\right) ; \underline{\mu}<\lambda<\bar{\mu}\right\},
$$


where $\mu:=\nu_{1}\left(\Omega_{+}\right)$. If (D1) (D3) are satisfied, then $\nu_{2}(\Omega)=\nu_{1}\left(\Omega_{+}\right)$. Thus $\Sigma_{+}$and $\Sigma_{-}$emanate from $\left(\nu_{2}(\Omega), 0\right)$. We obtain

Lemma 3.1. Suppose that (D1) $-(\overline{\mathrm{D} 3})$ and (F) hold. There exist two unbounded curves, which are $\Sigma_{ \pm}$defined by (3.5), consisting of nontrivial solutions to $\left(D P_{\Omega}\right)$ and emanating from the second eigenvalue $\left(\nu_{2}(\Omega), 0\right)$. Each curve is parametrized by $\lambda$. This bifurcation is supercritical.

Hereafter, we prove Theorem A. We have already constructed two unbounded curves $\Sigma_{ \pm}$emanating from the second eigenvalue which are parametrized by $\lambda$. It is clear that the closure of $\Sigma_{+} \cup \Sigma_{-}$is $\Sigma_{+} \cup \Sigma_{-} \cup\left\{\left(\nu_{2}(\Omega), 0\right)\right\}$ and homeomorphic to $\mathbb{R}$. Lemma 3.2 below is the main result of this section.

Lemma 3.2. Let $\Sigma_{+}$and $\Sigma_{-}$be defined by (3.5). Let $\left(\lambda_{0}, u^{*}\left(\lambda_{0}\right)\right) \in \Sigma_{+}$be fixed. Consider the eigenvalue problem

$$
\Delta \varphi+\lambda_{0} f^{\prime}\left(u^{*}\left(\lambda_{0}\right)\right) \varphi+\kappa \varphi=0 \quad \text { in } \Omega, \quad \varphi=0 \quad \text { on } \partial \Omega .
$$

Then the following hold:

(i) The first eigenvalue of (3.6) is (strictly) negative.

(ii) The second eigenvalue of (3.6) is (strictly) positive, and zero is not an eigenvalue.

Because of the nondegeneracy stated in Lemma 3.2 and the supercritical bifurcation at $\left(\nu_{2}(\Omega), 0\right)$, we see by the implicit function theorem that $\Sigma_{+} \cup \Sigma_{-} \cup\left\{\left(\nu_{2}(\Omega), 0\right)\right\}$ is the maximal continua of nontrivial solutions emanating from $\left(\nu_{2}(\Omega), 0\right)$. Theorem A immediately follows from Lemma 3.2

First, we prove (i) and (ii) in Lemma 3.2 when $(\lambda, u)$ is near the bifurcation point.

Lemma 3.3. If $\left(\lambda_{0}, u^{*}\left(\lambda_{0}\right)\right) \in \Sigma_{+}$is near $\left(\nu_{2}(\Omega), 0\right)$, then (i) and (ii) in Lemma 3.2 hold.

Proof. We briefly prove the lemma. We study eigenvalues of the operator $L:=\Delta+$ $\lambda_{0} f^{\prime}\left(u^{*}\right)$ on $\Omega$ with the Dirichlet boundary condition. When $\left(\lambda_{0}, u^{*}\right)=\left(\nu_{2}(\Omega), 0\right)$, the second eigenvalue is zero and simple. Since the bifurcation is supercritical, it follows from the principle of exchange of eigenvalues that the simple near-zero eigenvalue for a bifurcating solution near $\left(\nu_{2}(\Omega), 0\right)$, which is the second eigenvalue, is positive. Therefore (i) and (ii) hold, because of the continuity of each eigenvalue with respect to $\lambda$.

Next, we study the shape of $u \in \Sigma_{+} \cup \Sigma_{-}$. In this lemma we use the assumptions (D2) and (D3).

Lemma 3.4. Let $\left(\lambda_{0}, u^{*}\left(\lambda_{0}\right)\right) \in \Sigma_{+}$be fixed. Then the following hold:

(i) $u^{*}(x,-y)=u^{*}(x, y)$.

$$
\text { (ii) } u_{x}^{*}\left\{\begin{array} { l l } 
{ < 0 } & { \text { on } \partial \Omega \backslash T , } \\
{ = 0 } & { \text { on } \partial \Omega \cap T , }
\end{array} \quad \text { (iii) } u _ { \theta } ^ { * } \left\{\begin{array}{lll}
<0 & \text { on } & \Gamma_{+} \backslash T, \\
=0 & \text { on } & \partial \Omega \cap(S \cup T) \\
>0 & \text { on } & \Gamma_{-} \backslash T .
\end{array}\right.\right.
$$

(iv) $u_{x}^{*}(-x, y)=u_{x}^{*}(x, y), u_{x}^{*}(x,-y)=u_{x}^{*}(x, y)$.

(v) $u_{\theta}^{*}(-x, y)=u_{\theta}^{*}(x, y), u_{\theta}^{*}(x,-y)=-u_{\theta}^{*}(x, y)$. 
Proof. Since $u^{*}$ is a positive solution in $\Omega_{+}$, (i) follows from the celebrated symmetry result of Gidas-Ni-Nirenberg [5]. Here we used (D1) and (D2).

Let $\partial_{\nu}$ denote the outer normal derivative on $\partial \Omega$. Let

$$
\{(p(t), q(t))\}_{0 \leq t<L}(=\partial \Omega)
$$

be a parametrization of $\partial \Omega$ parametrized by the arc length such that $\partial_{\nu}=\dot{q} \partial_{x}-$ $\dot{p} \partial_{y}$. Here $\dot{p}:=p_{t}$. Then when $P \in \partial \Omega \cap T_{+}, \partial_{\nu} u^{*}\left(=\dot{q} u_{x}^{*}-\dot{p} u_{y}^{*}\right)<0$ at $P$ (Hopf's boundary point lemma) and $\dot{p} u_{x}^{*}+\dot{q} u_{y}^{*}=0$ at $P$ (the Dirichlet boundary condition). Because of (D2), $\dot{q}>0$ at $P$; in particular $\dot{q} \neq 0$. We have $u_{y}^{*}=-\dot{p} u_{x}^{*} / \dot{q}$. Substituting this into $\partial_{\nu} u^{*}$, we have

$$
0>\partial_{\nu} u^{*}=\dot{q} u_{x}^{*}-\dot{p} u_{y}^{*}=\left(\dot{p}^{2}+\dot{q}^{2}\right) u_{x}^{*} / \dot{q} \text { at } P,
$$

which indicates that $u_{x}^{*}<0$ at $P$. It follows from a similar argument that $u_{x}^{*}<0$ on $\partial \Omega \cap T_{-}$. We easily show that $u_{x}^{*}=0$ on $\partial \Omega \cap T$. Thus (ii) holds.

It is clear that $u_{\theta}^{*}=0$ on $\partial \Omega \cap(S \cup T)$. If $P \in \partial \Omega \cap\{x>0$ and $y>0\}$, then $u_{x}^{*}<0$ and $\dot{q}>0$ at $P$. Because of (D3),$p \dot{p}+q \dot{q}<0$ at $P$. Substituting $u_{y}^{*}=-\dot{p} u_{x}^{*} / \dot{q}$ into $u_{\theta}^{*}$, we have

$$
u_{\theta}^{*}=-q u_{x}^{*}+p u_{y}^{*}=-(p \dot{p}+q \dot{q}) u_{x}^{*} / \dot{q}<0 \text { at } P .
$$

We can similarly prove other inequalities. (iii) holds.

We can easily show that (iv) and (v) hold, using the oddness (resp. evenness) of $u^{*}$ in $x$ (resp. $y$ ). The proof is complete.

We are now in a position to prove Lemma 3.2 .

Proof of Lemma 3.2. We define $L:=\Delta+\lambda_{0} f^{\prime}\left(u^{*}\left(\lambda_{0}\right)\right)$ on $\Omega$ with the Dirichlet boundary condition. Let $\sigma$ denote the set of the eigenvalues of $L$. We define $\sigma^{e}:=\left\{\kappa \in \sigma\right.$; there is a nontrivial $\varphi \in X^{e}$ such that $(\kappa, \varphi)$ satisfies (3.6) $\}$ and $\sigma^{o}:=\left\{\kappa \in \sigma\right.$; there is a nontrivial $\varphi \in X^{o}$ such that $(\kappa, \varphi)$ satisfies (3.6) $\}$, where $X^{e}:=\{u \in X ; u(x,-y)=u(x, y)\}$ and $X^{o}:=\{u \in X ; u(x,-y)=-u(x, y)\}$. We divide the proof into four steps.

Step $1\left(\sigma=\sigma^{e} \cup \sigma^{o}\right)$. Let $(\kappa, \varphi)$ be an eigenpair. Since $u(x,-y)=u(x, y)$ (Lemma $3.4(\mathrm{i})), \varphi(x,-y)$ is also an eigenfunction. Let $\varphi^{e}(x, y):=\varphi(x, y)+$ $\varphi(x,-y)$ and $\varphi^{o}(x, y):=\varphi(x, y)-\varphi(x,-y)$. Then $\varphi^{e}(x, y)$ and $\varphi^{o}(x, y)$ satisfy (3.6). We see by contradiction that $\varphi^{e} \not \equiv 0$ or $\varphi^{o} \not \equiv 0$. Suppose the contrary. Adding two equalities, we have $\varphi \equiv 0$, which is a contradiction. Since $\varphi^{e} \not \equiv 0$ or $\varphi^{o} \not \equiv 0,\left(\kappa, \varphi^{e}\right)$ or $\left(\kappa, \varphi^{o}\right)$ is an (nontrivial) eigenpair, hence $\sigma \subset \sigma^{e} \cup \sigma^{o}$. It is clear that $\sigma \supset \sigma^{e} \cup \sigma^{o}$. Thus

$$
\sigma=\sigma^{e} \cup \sigma^{o} .
$$

Step 2. In this step we will show by contradiction that the second eigenvalue of (3.6) is not zero. Let $\varphi$ be a second eigenfunction. Then $\varphi$ satisfies

$$
\Delta \varphi+\lambda_{0} f^{\prime}\left(u^{*}\right) \varphi=0 \text { in } \Omega, \quad \varphi=0 \text { on } \partial \Omega .
$$

Let $\psi(x, y):=\varphi(x, y)-\varphi(-x, y)$. Then $\psi$ satisfies (3.8). If $\psi \not \equiv 0$, then $\psi$ is odd with respect to the $y$-axis. Since $\psi$ has exactly two nodal domains, $\psi$ does not change sign in $\Omega_{+}$. Hence zero is an eigenvalue of (3.3) with $u_{0}$ replaced by $u^{*}$. However, we see by the same argument as before that the first eigenvalue of (3.2) is positive. Thus $\psi \equiv 0$, which means that

$$
\varphi(x, y)=\varphi(-x, y) .
$$


We show by contradiction that $\varphi \in X^{e}$. Suppose the contrary, i.e., $\varphi \notin X^{e}$. Then $\varphi \in X^{o}$, because of (3.7). We define $\xi(x, y):=u_{\theta y}^{*}(0,0) \varphi(x, y)-\varphi_{y}(0,0) u_{\theta}^{*}(x, y)$. Note that $u^{*} \in C^{2}$. Then $\xi$ satisfies (3.8) and

$$
\xi(-x, y)=\xi(x, y), \quad \xi(x,-y)=-\xi(x, y) .
$$

We easily see by (3.10) and the definition of $\xi(x, y)$ that $\xi(0,0)=\xi_{x}(0,0)=\xi_{y}(0,0)$. By Corollary 2.2 we see that $\xi \equiv 0$ or $\{\xi=0\}$ has at least four curves near $(0,0)$. The former case does not occur because $u_{\theta}$ does not satisfy the Dirichlet boundary condition (Lemma 3.4 (iii)). We consider the latter case. Each curve meets another curve or hits the boundary. We consider two cases.

Case 1-1. If a curve meets another curve, then $\{\xi=0\}$ has at least two loops in $\Omega$ because of the symmetry (3.10). Moreover, there is an open set in $\Omega$ outside two loops. By $\omega_{1}, \omega_{2}$ we denote two (open) subdomains enclosed by the two loops. We define $\Phi:=\xi_{1}+c \xi_{2}$. Here

$$
\xi_{1}:=\left\{\begin{array}{ll}
\xi & \text { in } \omega_{1}, \\
0 & \text { in } \Omega \backslash \omega_{1},
\end{array} \xi_{2}:= \begin{cases}\xi_{2} & \text { in } \omega_{2}, \\
0 & \text { on } \Omega \backslash \omega_{2},\end{cases}\right.
$$

and $c$ is chosen such that $\left\langle\Phi, \Psi_{1}\right\rangle=0$, where $\Psi_{1}$ is a first eigenfunction of (3.6).

On the other hand, we define

$$
\mathcal{H}[\phi]:=\int_{\Omega}\left(|\nabla \phi|^{2}-\lambda_{0} f^{\prime}\left(u^{*}\right) \phi^{2}\right) d x .
$$

Then we have

$$
\begin{aligned}
\mathcal{H}[\Phi]= & \int_{\Omega}\left(|\nabla \Phi|^{2}-\lambda_{0} f^{\prime}\left(u^{*}\right) \Phi^{2}\right) d x \\
= & \int_{\omega_{1}}\left(\left|\nabla \xi_{1}\right|^{2}-\lambda_{0} f^{\prime}\left(u^{*}\right) \xi_{1}^{2}\right) d x+c^{2} \int_{\omega_{2}}\left(\left|\nabla \xi_{2}\right|^{2}-\lambda_{0} f^{\prime}\left(u^{*}\right) \xi_{2}^{2}\right) d x \\
& \quad+2 c \int_{\omega_{1} \cap \omega_{2}}\left(\nabla \xi_{1} \cdot \nabla \xi_{2}-\lambda_{0} f^{\prime}\left(u^{*}\right) \xi_{1} \xi_{2}\right) d x \\
= & -\int_{\omega_{1}} \xi_{1}\left(\Delta \xi_{1}+\lambda_{0} f^{\prime}\left(u^{*}\right) \xi_{1}\right) d x+\int_{\partial \omega_{1}} \xi_{1} \partial_{\nu} \xi_{1} d \sigma \\
& \quad-c^{2} \int_{\omega_{2}} \xi_{2}\left(\Delta \xi_{2}+\lambda_{0} f^{\prime}\left(u^{*}\right) \xi_{2}\right) d x+c^{2} \int_{\partial \omega_{2}} \xi_{2} \partial_{\nu} \xi_{2} d \sigma=0,
\end{aligned}
$$

where we use $\Delta \xi_{j}+\lambda_{0} f^{\prime}\left(u^{*}\right) \xi_{j}=0$ in $\omega_{j}(j=1,2)$ and $\omega_{1} \cap \omega_{2}=\emptyset$. It follows from a variational characterization of the second eigenvalue that

$$
\kappa_{2}=\inf _{\substack{\phi \in H^{1} \backslash\{0\} \\ \phi \in \operatorname{span}\left\{\Psi_{1}\right\rangle}} \frac{\mathcal{H}[\phi]}{\|\phi\|_{L^{2}}^{2}} \leq \frac{\mathcal{H}[\Phi]}{\|\Phi\|_{L^{2}}^{2}}=0 .
$$

If the equality holds, then $\Phi$ is a second eigenfunction. However, $\Phi=0$ on an open set in $\Omega$, which contradicts the unique continuation property. The above inequality is strict; hence $\kappa_{2}<0$. This contradicts the fact that the second eigenvalue is zero.

Case 1-2. If a curve hits a point $P$ on the boundary, then $\xi=0$ at $P$; hence $u_{\theta}^{*}=0$ at $P$. By Lemma 3.4 (iii) we see that $P \in \partial \Omega \cap(S \cup T)$. Because of the symmetry, $\{\xi=0\}$ has at least two loops in $\Omega$ and there is an open set in $\Omega$ outside two loops. Thus using the same argument as in Case 1-1, we obtain a contradiction. 
Two cases (Cases 1-1 and 1-2) are verified. The assumption, which is $\varphi \in X^{o}$, is not valid; hence $\varphi \in X^{e}$. We have

$$
\varphi(x,-y)=\varphi(x, y) \text {. }
$$

Differentiating (3.9) (resp. (3.11) ) with respect to $x$ (resp. $y$ ) and evaluating it at $(0,0)$, we have $\varphi_{x}(0,0)=-\varphi_{x}(0,0)$ (resp. $-\varphi_{y}(0,0)=\varphi_{y}(0,0)$ ). Thus $\varphi_{x}(0,0)=\varphi_{y}(0,0)=0$. We divide the possibilities into two cases.

Case 2-1. We consider the case $\varphi(0,0)=0$. Then $(0,0)$ is a degenerate zero of $\varphi$ of order at least two. Since $\varphi \not \equiv 0$, there are at least four curves of $\{\varphi=0\}$ emanating from $(0,0)$. Each curve meets another curve or hits the boundary. No matter which case occurs, $\varphi$ has at least three nodal domains because of the symmetries (3.9) and (3.11). This contradicts the fact that $\varphi$ is a second eigenfunction, because $\varphi$ should have exactly two nodal domains.

Case 2-2. We consider the case $\varphi(0,0) \neq 0$. We define $\eta(x, y):=u_{x}^{*}(x, y)$. Since $\Delta u^{*}+V\left(u^{*}\right) u^{*}=0$ in $\Omega_{+}, u^{*}=0$ on $\partial \Omega_{+}$and $u^{*}>0$ in $\Omega_{+}$, we see by Hopf's lemma that $\eta(0,0)\left(=u_{x}^{*}(0,0)\right)>0$. Lemma 3.4 (i) says that

$$
u^{*}(x,-y)=u^{*}(x, y) \text {. }
$$

Differentiating (3.12) with respect to $x$ and $y$ and evaluating it at $(0,0)$, we have $-u_{x y}^{*}(0,0)=u_{x y}^{*}(0,0)$ which indicates $\eta_{y}(0,0)\left(=u_{x y}^{*}(0,0)\right)=0$. Differentiating $u^{*}(-x, y)=-u^{*}(x, y)$ twice with respect to $x$ and evaluating it at $(0,0)$, we have $\eta_{x}(0,0)\left(=\eta_{x x}(0,0)\right)=0$. We define $\zeta(x, y):=\varphi(0,0) \eta(x, y)-\eta(0,0) \varphi(x, y)$. Then $\zeta(-x, y)=\zeta(x, y)=\zeta(x,-y)$ (Lemma 3.4 (iv)) and $\zeta(0,0)=\zeta_{x}(0,0)=\zeta_{y}(0,0)=$ 0 . Since $\zeta$ satisfies $\Delta \zeta+\lambda_{0} f^{\prime}\left(u^{*}\right) \zeta=0$, it follows from Corollary 2.2 that $\zeta \equiv 0$ or $\{\zeta=0\}$ has at least four curves emanating from $(0,0)$. Since $\eta\left(=u_{x}^{*}\right)$ does not satisfy the Dirichlet boundary condition (Lemma 3.4 (ii)), $\zeta \not \equiv 0$. If a curve meets another curve, then $\{\zeta=0\}$ has at least two loops. If a curve hits a point on the boundary, then $\zeta=0$ at the point; hence the point is on $\partial \Omega \cap T$ (Lemma 3.4 (ii)). Because of the symmetries, $\{\zeta=0\}$ has at least two loops. No matter which case occurs, $\{\zeta=0\}$ has two loops and there is an open set in $\Omega$ outside two loops. By the same argument as in Case 1-1 we obtain a contradiction.

Two cases (Cases 2-1 and 2-2) are verified. We have shown that zero is not the second eigenvalue of (3.6).

Step 3. In this step we will show by contradiction that the first eigenvalue of (3.6) is not zero. Let $\varphi$ be a corresponding eigenfunction. Using the same argument with $\zeta$ as in Case 2-2, we can obtain a contradiction. We omit the details.

Step 4. When $\left(\lambda_{0}, u^{*}\left(\lambda_{0}\right)\right)$ is near $\left(\nu_{2}(\Omega), 0\right)$, statements (i) and (ii) follow from Lemma 3.3. Combining the continuity of each eigenvalue with respect to $\lambda$ and the results of Steps 2 and 3, we see that (i) and (ii) hold for every $\left(\lambda_{0}, u^{*}\left(\lambda_{0}\right)\right) \in \Sigma_{+}$. Otherwise, there is $\left(\lambda_{1}, u^{*}\left(\lambda_{1}\right)\right) \in \Sigma_{+}$such that zero is the first or second eigenvalue, which contradicts Step 2 or Step 3.

\section{REFERENCES}

[1] T. Carleman, Sur les systèmes linéaires aux derivées partielles du premier ordre à deux variables, C. R. Acad. Sci. Paris 197 (1933), 471-474.

[2] N. Chafee and E. Infante, A bifurcation problem for a nonlinear partial differential equation of parabolic type, Applicable Anal. 4 (1974/75), 17-37. MR0440205 (55:13084) 
[3] M. Crandall and P. Rabinowitz, Bifurcation from simple eigenvalues, J. Funct. Anal. 8 (1971), 321-340. MR0288640 (44:5836)

[4] M. del Pino, J. García-Melián and M. Musso, Local bifurcation from the second eigenvalue of the Laplacian in a square, Proc. Amer. Math. Soc. 131 (2003), 3499-3505. MR1991761 (2004c:35027)

[5] B. Gidas, W.-M. Ni and L. Nirenberg, Symmetry and related properties via the maximum principle, Comm. Math. Phys. 68 (1979), 209-243. MR544879 (80h:35043)

[6] M. Holzmann and H. Kielhöfer, Uniqueness of global positive solution branches of nonlinear elliptic problems, Math. Ann. 300 (1994), 221-241. MR1299061 (95m:35068)

[7] P. Hartman and A. Wintner, On the local behavior of solutions of non-parabolic partial differential equations, Amer. J. Math. 75 (1953), 449-476. MR0058082 (15:318b)

[8] P. Korman, Solution curves for semilinear equations on a ball, Proc. Amer. Math. Soc. 125 (1997), 1997-2005. MR1423311 (97j:35037)

[9] Y. Miyamoto, Global branches of non-radially symmetric solutions to a semilinear Neumann problem in a disk, J. Funct. Anal. 256 (2009), 747-776. MR2484935 (2010d:35130)

[10] Y. Miyamoto, Non-existence of a secondary bifurcation point for a semilinear elliptic problem in the presence of symmetry, J. Math. Anal. Appl. 357 (2009), 89-97. MR 2526808 (2010d:35131)

[11] Y. Miyamoto, Global branches of sign-changing solutions to a semilinear Dirichlet problem in a disk, preprint.

[12] Y. Mukai, Bifurcation of solutions to a boundary value problem of a semilinear elliptic equation on a disk (in Japanese), Master Thesis (1997), Graduate School of Mathematical Sciences, University of Tokyo, Japan.

[13] R. Pütter, On the nodal lines of second eigenfunctions of the fixed membrane problem, Comment. Math. Helv. 65 (1990), 96-103. MR.1036131 (91c:35109)

[14] C. Shen, Remarks on the second eigenvalue of a symmetric simply connected plane region, SIAM J. Math. Anal. 19 (1988), 167-171. MR924553 (89a:35152)

[15] J. Vegas, Bifurcations caused by perturbing the domain in an elliptic equation, J. Differential Equations 48 (1983), 189-226. MR696867 (84g:35020)

Department of Mathematics, Tokyo Institute of Technology, Meguro-ku, Tokyo 152-8551, JAPAN

E-mail address: miyamoto@math.titech.ac.jp 